\title{
IAMJ
}

INTERNATIONAL

AYURVEDIC

MEDICAL JOURNAL

\section{UNUSUAL ANIMAL RESOURCES EXPLAINED IN RASA RATNA SAMUCCHAYA}

\author{
Devika M. B ${ }^{1}$, M. S. Krishnamurthy ${ }^{2}$ \\ ${ }^{1}$ PG Scholar, ${ }^{2}$ Professor and HOD; \\ Dept. of PG studies in Rasashastra and Bhaishajya Kalpana, Alva's Ayurveda Medical College, Moodubidire, \\ Karnataka, India
}

Corresponding Author: nayana.raj1212@gmail.com

\section{https://doi.org/10.46607/iamj2508082020}

(Published online: August 2020)

Open Access

(C) International Ayurvedic Medical Journal, India 2020

Article Received:27/07/2020 - Peer Reviewed:05/08/2020 - Accepted for Publication:05/08/2020

Check for updates

\section{ABSTRACT}

Rasa Ratna Samucchaya being an important treatise of Rasashastra from $12^{\text {th }}$ Century A.D consists of various references regarding the usage of diverse animal resources. Resources like various products and body parts by different animals are derived and are used for various purposes in different context. They were used for the purpose of Shodhana (purification), Marana (incineration), Satwapatana (process of extraction of chief constituent of any mineral), Druti nirmana (liquid form of any metal/mineral) and in preparation of different Mushas (crucibles) like Vajra musha, Vajra dravanimusha, Garamusha etc., as well as in the preparation of Rasayogas (formulations). They are also used as Anupana/Sahapana (adjuvants), Pathya and Apathya (diet and regimens to be followed and avoided). Not only animal resources but utilization of resources of birds, insects, worm as well as aquatic resources like Pravala (coral), Mukta (pearl), Shankha (conch shell), Muktashukti (pearl oyster shell), Agnijara (ambergies) etc., were also explained. In addition, usage of Nara mutra (human urine), Narasthi (human bone) and Nari sthanya (breast milk) are also mentioned. This article is an effort to compile and enlist about the assorted animal resources that has been told in Ayurveda Rasashastric text Rasa ratna samucchaya written by Rasa Vagbhata.

Keywords: Rasa Ratna Samucchaya, Animal resources, Rasayogas 


\section{INTRODUCTION}

In Ayurveda, there are numerous references regarding the utility of animal resources in various contexts. Similarly, in Rasa Ratna Samucchaya (RRS), wide varieties of animals, birds and insects etc., resources are used in the preparation of formulations for the treatment of various diseases. Many of the animal resources are included in the classification of Rasas and they are extensively used in various preparations. Kankusta which is considered as Varchas (faeces) of newborn horse/Nabinala (umbilical cord) of horse is classified under Uparasas $^{1}$. Similarly, Kaparda/Varatika (covries - outer shell of an aquatic animal Cypereamoneta Linn.) and Agnijara (placental part of a variety of fish/ambergies) are classified under Sadharana rasas ${ }^{2}$. Pravalal Abdhijantu (coral - a skeleton of sea animal Corallium rubrum Linn.), Mukta (pearl - a secretion produced by various mollusks) are classified under Ratnavarga ${ }^{3}$ (group of precious stones) and Shankha (conch - outer shell of molluscan TurbinellarapaLamarck), Sukti/Muktasukti (pearl oyster - outer shell of aquatic animal Ostrae edulis Linn.) are also used in different formulations. While classifying the Rasa dravyas, Acharya Vagbhata has given many similes which are named by correlating the body parts as well as sounds and smell of the different animals/birds for easy understanding e.g. Nagabhra, Mandukabhra ${ }^{4}$ (Similar to the sound produced by them on heating the metal) in the context of types of Abhraka, Mayurakanta sadrusha 5 (similar to peacock's neck colour) while explaining about Grahyalakshana (good variety) of Vaikranta, Gomutragandhi shilajatu $^{6}$ (similar to smell of cow's urine) as a type of Shilajatu etc. Different Putas (temperature pattern) like Gaja (elephant) puta, Kapota (pigeon) puta ${ }^{7}$ etc., are named after different animals/birds name based on the number of cow dung cakes used. Different Vargas (group of drugs) which includes animal resources is also mentioned in this text like Vasadivarga, Mutra varga, Dugdhavarga, Mahishapanchaka, Vit varga and Rakta varga ${ }^{8}$.The usage of such commonly used animal resources as well as rarely used and practiced animal resources in the pharmaceutical preparations are presented in Table No.1. Even though cow deriva- tives like milk, curd, butter, buttermilk, cow dung etc are quoted in several occasions quite interestingly several other animal products are also used. However, goat, buffalo, sheep, horse etc. are commonly found and their products or derivatives are used. In addition, few rare and uncommon animal resources are also in practice. They are compiled together and presented in Table No. 2. Likewise, few of the unique parts of animals, birds, insects as well as aquatic animals are used directly in the treatment like external application, potentiating, detoxification etc. Even though such uncommon, non-universally acceptable practices are beyond the scope of time, it needs to be documented as the historical evidences give life for few of the subjects and practices. Table No. 3 denotes unique body parts of animals, birds, insects as well as aquatic animals used directly or indirectly in the treatment. Few unique formulations which are prepared by using various animal products are explained below:

\section{Vajra/Heerakamarana (Incineration of dia-} mond): Method I 9: Shodhita vajra (Purified diamond) is triturated with Matkuna rakta (Bed bug's blood) for 4 times. Later it is wrapped with the Chucchundara mamsa (Rat's flesh) and subjected to Varahaputa. Like this it should be given with 30 Varahaputas to get Heeraka bhasma. Method II $^{\mathbf{1 0}}$ : In another method, Bhunaga (Earthworm) is triturated with Madanaphala kashaya (Decoction of Randia dumetorum Lam.) this paste is applied to Heeraka (Diamond). After complete drying it is subjected to 20 Gajaputa to obtain Vajra bhasma.

2. Pittala druti ${ }^{11}$ : Pittala churna (Brass powder) is mixed with any food preparations and fed to black colored goat. The faeces obtained from this goat are burnt into ashes and it is used as Pittala druti, which is having Rasa rasayana quality.

3. Maheshwara dhoopa ${ }^{12}$ : Srivesta (Soot/Carbon black), Devadaru - Cedrus deodara Roxb., Bahlika (Hingu) - Ferulanorthax Bioss, Musta Cyperusrotundus Linn., Katukarohini - Picrorhizakurroa Royle ex Benth., Sarsapa - Brassica campestris Linn., Nimbapatra - Azadirachta indi- 
ca A. Juss, Madanaphala - Randia dumetorum Lam., Vacha - Acorus calamus Linn., BhruhatiSolanum indicum Linn., Kantakari - Solanum xanthocarpum Brum., Sarpa nirmoka (Snake's skin - Ecdysis), Karpasa beeja - Gossypium herbaceum Linn., Yavasthusha (Barley husk), Goshrunga (Horn of cow), Khara roma (Rabbit's hair), Barhi piccha (Peacock's feather), Vidala vit (Cat's feaces), Chaga roma (Goat's hair), Cow's ghee are triturated with Sheep's urine. And this is used as Dhoopa (Fumigation) in Sarva graharoga.

4. Grahaghna dhoopa ${ }^{13}$ : Karpasa beeja - Gossypium herbaceum Linn., Mayura piccha (Peacock's feather), Bhruhati - Solanum indicum Linn., Nirmalya, Pinditaka (Madanaphala) - Randia dumetorum Lam., Twak - Cinnamomum zeylenica Blume, Jatamansi - Nardostachys jatamansi Dc., Vrusha (Vasa) - Adathoda vasicaNees., Damsa vit (Cat's feaces), Tusha, Vacha - Acorus calamus Linn., Keshahi nirmoka (Donkey's hair), Nagendra (Snake), Dwija shrunga (Elephant tusk), Hingu - Ferula northax Bioss, Maricha-Piper nigrum Linn. are used as Dhoopa in Skandhonmada and Graharoga.

5. Narasthi taila ${ }^{\mathbf{1 4}}$ : Narasthi (Human bone) is made into powder and Kalka (Paste) is prepared by mixing in water. Later 4 parts of Tila taila (Sesame oil - Sesamum indicum Linn.) and 16 parts of water is added to the above Kalka. This mixture is subjected to heat till it attains Taila siddhi lakshanas ${ }^{15}$ and the obtained Taila is used for external application in Bhagandara (Fisula-in-ano).

6. Chucchundari taila ${ }^{16}$ : Chucchundara mamsa (Rat's flesh) is made into Kalka (Paste). Later 4 parts of Tila taila (Sesame oil - Sesamum indicum Linn.) and 16 parts of water is added to the above Kalka. This mixture is subjected to heat till it attains Taila siddhi lakshanas and the obtained Taila is used for external application in Gandamala (Goiter).

\section{DISCUSSION}

This text emphasizes on the significant usage of animal resources along with herbal and mineral drug resources. Animal resources are used in almost all the varieties of Kalpana. Not only animal resources but utilization of resources of birds, insects and worm as well as aquatic resources is also told. Vargas (Group of drugs) like Mutra varga, Vit varga, Raktavarga etc are mentioned which extensively consists of distinct animal products. Many unique and rare preparations are explained by using various animal products which are used both internally as well as externally in different disease conditions. They are not only used in the preparation as an ingredient but also used for the purpose of Shodhana (Purification), Marana (Incineration) etc procedures during drug processing, as Anupana/Sahapana (Adjuvants), Pathya and Apathya (Diet and regimens to be followed and avoided). During Puta (Incineration carried out by maintaining specific temperature pattern) cow dung cakes are the main fuel used to regulate the temperature. This will help to procure the properly formed medicines. Among all animal resources predominant usage of various products of cow can be noticed throughout the textbook. This shows the abundance of animal resources during those days.

\section{CONCLUSION}

Therapeutic efficacy of the many preparations should be explored scientifically e.g. Shunoasthi nasya, $\mathrm{Na}$ rasthi taila etc., for their better practical applicability. As many of the animal resources are not available nowadays, suitable substitutes can be evolved for the fulfillment of therapeutic and pharmaceutical needs. In spite of enormous classical references regarding the utility of the animal resource, their practical employment is questionable because of the ethical issues.

\section{REFERENCES}

1. Acharya Vagbhata, Rasa Ratna Samucchaya, Siddhiprada Hindi Commentary By Mishra Siddhinandan, Chaukhambha Orientalia, Varanasi, First Edition 2011, Chapter 3, Slk No. 1, Pg. No. 60

2. Acharya Vagbhata, Rasa Ratna Samucchaya, Siddhiprada Hindi Commentary By Mishra Siddhinandan, 
Chaukhambha Orientalia, Varanasi, First Edition 2011, Chapter 3, Slk. No. 123-124 Pg. No. 85

3. Acharya Vagbhata, Rasa Ratna Samucchaya, Siddhiprada Hindi Commentary By Mishra Siddhinandan, Chaukhambha Orientalia, Varanasi, First Edition 2011, Chapter 4, Slk. No. 6, Pg. No. 96

4. Acharya Vagbhata, Rasa Ratna Samucchaya, Siddhiprada Hindi Commentary By Mishra Siddhinandan, Chaukhambha Orientalia, Varanasi, First Edition 2011, Chapter 2, Slk. No. 4, Pg. No. 30

5. Acharya Vagbhata, Rasa Ratna Samucchaya, Siddhiprada Hindi Commentary By Mishra Siddhinandan, Chaukhambha Orientalia, Varanasi, First Edition 2011, Chapter 2, Slk. No. 56 Pg. No. 37

6. Acharya Vagbhata, Rasa Ratna Samucchaya, Siddhiprada Hindi Commentary By Mishra Siddhinandan, Chaukhambha Orientalia, Varanasi, First Edition 2011, Chapter 2, Slk. No. 103, Pg. No. 47

7. Acharya Vagbhata, Rasa Ratna Samucchaya, Siddhiprada Hindi Commentary By Mishra Siddhinandan, Chaukhambha Orientalia, Varanasi, First Edition 2011, Chapter 10, Slk. No. 53 And 55, Pg. No. 243

8. Acharya Vagbhata, Rasa Ratna Samucchaya, Siddhiprada Hindi Commentary By Mishra Siddhinandan, Chaukhambha Orientalia, Varanasi, First Edition 2011, Chapter 10, Slk. No. 74-76, 81, 85-89, Pg. No. 246-248

9. Acharya Vagbhata, Rasa Ratna Samucchaya, Siddhiprada Hindi Commentary By Mishra Siddhinandan,
Chaukhambha Orientalia, Varanasi, First Edition 2011, Chapter 4, Slk. No. 36-37, Pg. No. 118

10. Acharya Vagbhata, Rasa Ratna Samucchaya, Siddhiprada Hindi Commentary By Mishra Siddhinandan, Chaukhambha Orientalia, Varanasi, First Edition 2011, Chapter 4, Slk. No. 44-45, Pg. No. 119

11. Acharya Vagbhata, Rasa Ratna Samucchaya, Siddhiprada Hindi Commentary By Mishra Siddhinandan, Chaukhambha Orientalia, Varanasi, First Edition 2011, Chapter 5, Slk. No. 202-203, Pg. No. 186

12. Acharya Vagbhata, Rasa Ratna Samucchaya, Siddhiprada Hindi Commentary By Mishra Siddhinandan, Chaukhambha Orientalia, Varanasi, First Edition 2011, Chapter 22, Slk. No. 163-165, Pg. No. 539

13. Acharya Vagbhata, Rasa Ratna Samucchaya, Siddhiprada Hindi Commentary By Mishra Siddhinandan, Chaukhambha Orientalia, Varanasi, First Edition 2011, Chapter 23,Slk. No. 16, Pg. No. 544

14. Acharya Vagbhata, Rasa Ratna Samucchaya, Siddhiprada Hindi Commentary By Mishra Siddhinandan, Chaukhambha Orientalia, Varanasi, First Edition 2011, Chapter 24, Slk. No. 118, Pg. No. 578

15. Srivatsava Shailaja, Sharangadhara Samhita, Jiwanprada Hindi Commentary, Chaukhambha Orientalia, Varanasi, Reprint Edition: 2011, Chapter 9, Slk. No. 13-14, Pg. No. 217

16. Acharya Vagbhata, Rasa Ratna Samucchaya, Siddhiprada Hindi Commentary By Mishra Siddhinandan, Chaukhambha Orientalia, Varanasi, First Edition 2011, Chapter 24, Slk. No. 141, Pg. No. 582

\section{$\underline{\text { Tables }}$}

Table 1: Usage of cow's ghee/milk/butter/buttermilk/curd/urine/dung/fat/bile in various contexts

\begin{tabular}{|c|c|c|c|}
\hline $\begin{array}{l}\text { For preparation as ingre- } \\
\text { dient }\end{array}$ & For Shodhana/Marana/Satwapatana & As Anupana/Sahapana & As Pathya and Apathya \\
\hline Deepika rasa & $\begin{array}{l}\text { Abhraka shodhana, satwapatana, } \\
\text { satwashodhana }\end{array}$ & Parpati rasa & Lokanatha rasa \\
\hline Chandrakala rasa & Vaikranta shodhana and satwapatana & Kanasundara rasa & Ananda bhairava rasa \\
\hline Swasarivataka & Swarnamakshika satwapatana & Lokeshwara rasa & Rasottama rasa \\
\hline Kanaka sundara rasa & Shilajatu shodhana & Agnikumara rasa & Lokeshwara rasa \\
\hline Panchamrutaparpati & Sasyaka shodhana & Laghusiddhabhra rasa & Agnikumara rasa \\
\hline Arsakutara rasa & Karpara satwa & Grahanikapata rasa & $\begin{array}{l}\text { Kanakasundara rasa (In } \\
\text { grahani) }\end{array}$ \\
\hline Mulakutara rasa & Gandhaka shodhana & Vaishwanarapottali rasa & Grahanihara rasa \\
\hline $\begin{array}{l}\text { Laghulokanatheshwara } \\
\text { rasa }\end{array}$ & Gairika shodhana & Kravyada rasa & Grahanikapata rasa \\
\hline Vasantakusumakara rasa & Spatika satwapatana & Rakshasa rasa & Vidhwamsa rasa \\
\hline
\end{tabular}




\begin{tabular}{|c|c|c|c|}
\hline Darvi churna & Parada bandhana in Anjana & Pramehari rasa & $\begin{array}{l}\text { In vaishwanarapottali } \\
\text { rasa-Ghrita, takra }\end{array}$ \\
\hline Gomutradhya taila & Rajavarta shodhana & Mehashatru rasa & Vangeshwara rasa \\
\hline $\begin{array}{l}\text { Gandhakadipottali rasa } \\
\text { (1) and (2) }\end{array}$ & Dhatu samanya shodhana & Kaseesabaddha rasa & Pramehari rasa \\
\hline Agni kumara rasa (1) & Lohavishesha shodhana & Sanjeevana rasa & Uma shambu rasa \\
\hline Takrasava & Hingu shodhana & Mehakutara rasa & Panchanana rasa \\
\hline Shambukadi loha & Loha marana & Mehabaddha rasa & Vijaya rasa/gutika \\
\hline Purnachandra rasa & Rasa marana & Vangeshwara rasa & Khageswara rasa \\
\hline Kushmanda khanda & & Vidyadhara rasa & Dhanwantari rasa \\
\hline Trailokyasundara rasa & Miscellaneous & Vaishwanara rasa & Ankoladi lepa \\
\hline Hamsamandura & Garamusha & Vyoshadi churna & $\begin{array}{l}\text { Gandhashma garbharasa } \\
\text { (1) and (2) }\end{array}$ \\
\hline Devadalichurna & Mutravarga & Soubhagya yoga & Arka murti rasa \\
\hline Visarpanashanataila & Panchamruta & Agni kumara rasa & $\begin{array}{l}\text { Dhattura panchanga } \\
\text { ghrita }\end{array}$ \\
\hline Kustanashana rasa & Dugdavarga & Trinetra rasa & Triphala rasayana (4) \\
\hline Nagarjuna vati & Vid varga & Amrutarnava rasa & Sutendra rasa \\
\hline Talakeswara rasa & $\begin{array}{l}\text { In Mrughanka pottali rasa for making } \\
\text { Musha }\end{array}$ & Pitta rogantaka rasa & Kamadeepana rasa \\
\hline Medinisara rasa & & Vinodavidyadhara rasa & $\begin{array}{l}\text { Rasa sevanapathya - } \\
\text { Dadhi }\end{array}$ \\
\hline Swarnaksheeri rasa & & Mahavahni rasa & $\begin{array}{l}\text { Masanumasika garbhin- } \\
\text { ipathya }\end{array}$ \\
\hline & & & $\begin{array}{l}\text { Dhattura panchanga } \\
\text { ghrita }\end{array}$ \\
\hline Chandraprabha vatika & & Vajra kshara & As Apathya \\
\hline Udayadithya rasa & & Hamsa mandura & $\begin{array}{l}\text { In Vaishwanarapottali } \\
\text { rasa-Dadhi }\end{array}$ \\
\hline Nirgundyadilepa & & Kalavidhwamsana rasa & $\begin{array}{l}\text { Talakeswara rasa - Tak- } \\
\text { ra }\end{array}$ \\
\hline Krimishoolasamhara rasa & & Trailokyasundara rasa & $\begin{array}{l}\text { Parada jarananishiddha } \\
\text { - Gomutra }\end{array}$ \\
\hline $\begin{array}{l}\text { Gandhashmagarbha rasa } \\
\text { (2) }\end{array}$ & & Kameshwara rasa & \\
\hline Yogaraja guggulu (2) & & Sindurabhusana rasa & \\
\hline Shatavaryadi guggulu & & Taleswara rasa & \\
\hline Shadanga guggulu & & $\begin{array}{l}\text { Vajra shekara rasa (Kush- } \\
\text { ta) }\end{array}$ & \\
\hline Triphala yamaka & & Medinisara rasa & \\
\hline Eleya sarpi & & Switrari rasa & \\
\hline Eleyaka taila & & Kilasanashana rasa & \\
\hline Ashwatha ksheerapaka & & Krimishoolasamhara rasa & \\
\hline Madhukadi kalka & & Sheetari rasa & \\
\hline Yastyadi ghrita & & Martandeshwara rasa & \\
\hline Aswagandha ghrita & & Sarvavatari rasa & \\
\hline Nimbadi ghrita/ksheera & & Chandravaleha & \\
\hline
\end{tabular}




\begin{tabular}{|c|c|c|c|}
\hline & Laghulokeshwara rasa & Jalouka bandha & \\
\hline & Visarpanashana taila & Haratala satwapatana & \\
\hline & Kaphakustahara rasa & & \\
\hline & Kanaka sundara rasa & & \\
\hline & Tripurantaka rasa & & \\
\hline & Grahanashini gutika & & \\
\hline & Maheshwara dhoopa & & \\
\hline & Navanetradatri varti & & \\
\hline & Haridradi lepa & & \\
\hline & Vajrikantapashana lepa & & \\
\hline & Lakshadi prayoga & & \\
\hline & Mahabhra satwabhasma & & \\
\hline & Sindura rasa & & \\
\hline & Chandanadi taila & & \\
\hline & Timiravisha kalpa & & \\
\hline & Navajwaravisha kalpa & & \\
\hline Avi/Bastaksheera (Sheep's milk) & Maheshwara dhoopa & & Gokshuradi churna \\
\hline & $\begin{array}{l}\text { Vicharchikadi visha- } \\
\text { kalpa }\end{array}$ & & \\
\hline & Karnaamayaghna taila & & \\
\hline $\begin{array}{l}\text { Mahisha } \\
\text { urine/milk/buttermilk }\end{array}$ & Vishnukranta prayoga & Abhraka satwapatana & $\begin{array}{l}\text { Mahataleshwara rasa - } \\
\text { Ghee }\end{array}$ \\
\hline & Tamradruti (1) & Sasyaka shodhana & $\begin{array}{l}\text { Lohakalpa (5) - Butter- } \\
\text { milk }\end{array}$ \\
\hline & Mandura lepa & $\begin{array}{l}\text { Haratala shodhana and sat- } \\
\text { wapatana }\end{array}$ & \\
\hline & Others & & \\
\hline & Vajra dravani musha & & \\
\hline & Mahisha panchaka & & \\
\hline Haya $\quad$ (Horse) & Vajra musha & Vaikranta shodhana & \\
\hline urine/milk/buttermilk/saliva & $\begin{array}{l}\text { Swarna druti - Saliva of } \\
\text { horse }\end{array}$ & Vartaloha shodhana & \\
\hline & Arshoghna vatakam & Karpara shodhana & \\
\hline Nara mutra (Human urine) & Kanaka sundara rasa & Karpara shodhana & \\
\hline & Aparajadi nasya & & \\
\hline & Shunoasthi nasya & & \\
\hline & Sarpavisha chitiksa & & \\
\hline Nari sthanya (Breast milk) & Chinnadi taila & Loha marana & \\
\hline & Nrukapalanjana & & \\
\hline & Tamra druti (1) & & \\
\hline Nara asthi (Human bone) & Apasmara chikitsa & & \\
\hline & Nara asthi taila & & \\
\hline
\end{tabular}

Table 3: Usage of different animals, birds, insects as well as aquatic animal resources

\begin{tabular}{|l|l|l|l|} 
Resources used & $\begin{array}{l}\text { For preparation as ingre- } \\
\text { dient }\end{array}$ & $\begin{array}{l}\text { For } \\
\text { dhana/Marana/Satwapatana }\end{array}$ & $\begin{array}{l}\text { As Pathya and Ap- } \\
\text { athya }\end{array}$ \\
\hline Shasharakta (Rabbit's blood) & Shasharudhira prayoga in & Vishesha shodhana of loha &
\end{tabular}




\begin{tabular}{|c|c|c|c|}
\hline & vyanga & & \\
\hline & Bhutala prayoga & & \\
\hline Matkunarakta (Bed bug's blood) & & Vajra marana $(1)$ & \\
\hline \multirow[t]{2}{*}{ Bhunaga (Earth worm) } & Ratnakaranda rasa & Vajra marana (5) & \\
\hline & $\begin{array}{l}\text { Bhutalaprayoga in Bha- } \\
\text { gandaravrana chikitsa }\end{array}$ & & \\
\hline $\begin{array}{l}\text { Mushika/Chucchundara mamsa } \\
\text { (Rat's flesh) }\end{array}$ & Chucchundari taila & Vajra marana & \\
\hline \multirow[t]{2}{*}{ Indragopa (Rain bug) } & Vaikranta druti & & \\
\hline & Swarna druti & & \\
\hline $\begin{array}{l}\text { Sarasa (Sarus crane) rakta and } \\
\text { Sarasa mamsa }\end{array}$ & Yavaksharadilepa & & Amrutarnava rasa \\
\hline \multirow{4}{*}{$\begin{array}{l}\text { Shambuka mamsa (Snail and it's } \\
\text { shell) }\end{array}$} & Karshyahara yoga traya & & Kamadeepana rasa \\
\hline & Lohakalpa (8) & & \\
\hline & Pushpaharanjana & & \\
\hline & Shambukadi varti & & \\
\hline $\begin{array}{l}\text { Manduka asthi and Vasa (Bone and } \\
\text { fat of frog) }\end{array}$ & Swarna druti & & \\
\hline \multirow[t]{2}{*}{$\begin{array}{l}\text { Kukkuta Mamsa (Chicken)/Anda } \\
\text { twak/rasa (Eggshell/egg white) }\end{array}$} & Raktapittankusha rasa & & $\begin{array}{l}\text { Panchamruta parpati } \\
\text { - Kukkutanda mamsa } \\
\text { is Apathya }\end{array}$ \\
\hline & $\begin{array}{l}\text { Antravruddhihara } \\
\text { taila/ghrita }\end{array}$ & & \\
\hline Charmachetarakta & $\begin{array}{l}\text { Charmacheta prayoga } \\
\text { (Karnapalichikitsa) }\end{array}$ & & \\
\hline \multirow[t]{2}{*}{$\begin{array}{l}\text { Varaha vasa (Pig's fat) and } \\
\text { Varahakarnarakta (Pig's blood } \\
\text { from ear) }\end{array}$} & $\begin{array}{l}\text { Varaha vasa taila (In } \\
\text { karnapali chikitsa as le- } \\
\text { pa) }\end{array}$ & & \\
\hline & Brahmyadighrita nasya & & \\
\hline $\begin{array}{l}\text { Mayura mamsa and piccha (Pea- } \\
\text { cock's feather and flesh) }\end{array}$ & $\begin{array}{l}\text { Antravruddhi chikitsa } \\
\text { Grahaghna dhoopa - } \\
\text { Mayurapiccha }\end{array}$ & & \\
\hline $\begin{array}{l}\text { Tittira (Partridge) and Vartaka } \\
\text { (Quail) mamsa rasa }\end{array}$ & Antravruddhi chikitsa & & \\
\hline \multirow[t]{3}{*}{ Marjara $(\mathrm{Cat})$} & $\begin{array}{l}\text { Kanaka sundara rasa - } \\
\text { Cat's faeces is used }\end{array}$ & & \\
\hline & Grahaghna dhoopa & & \\
\hline & $\begin{array}{lr}\text { Marjarasthi } & \text { pralepa in } \\
\text { Bhagandara } & \text { vra- } \\
\text { nachikitsa } & \end{array}$ & & \\
\hline Harini shrunga (Hart's horn) & Lohakalpa (5) & & \\
\hline \multirow{3}{*}{$\begin{array}{l}\text { Shwana/Kukkura asthi (Dog's } \\
\text { bone) }\end{array}$} & Tilaja deepananjana & & \\
\hline & Shunoasthi nasya & & \\
\hline & $\begin{array}{lr}\text { Kukkurasthi } & \text { prayoga in } \\
\text { Bhagandara } & \text { vra- } \\
\text { nachikitsa } & \end{array}$ & & \\
\hline Krukalasa & Krukalasa prayoga & & \\
\hline
\end{tabular}




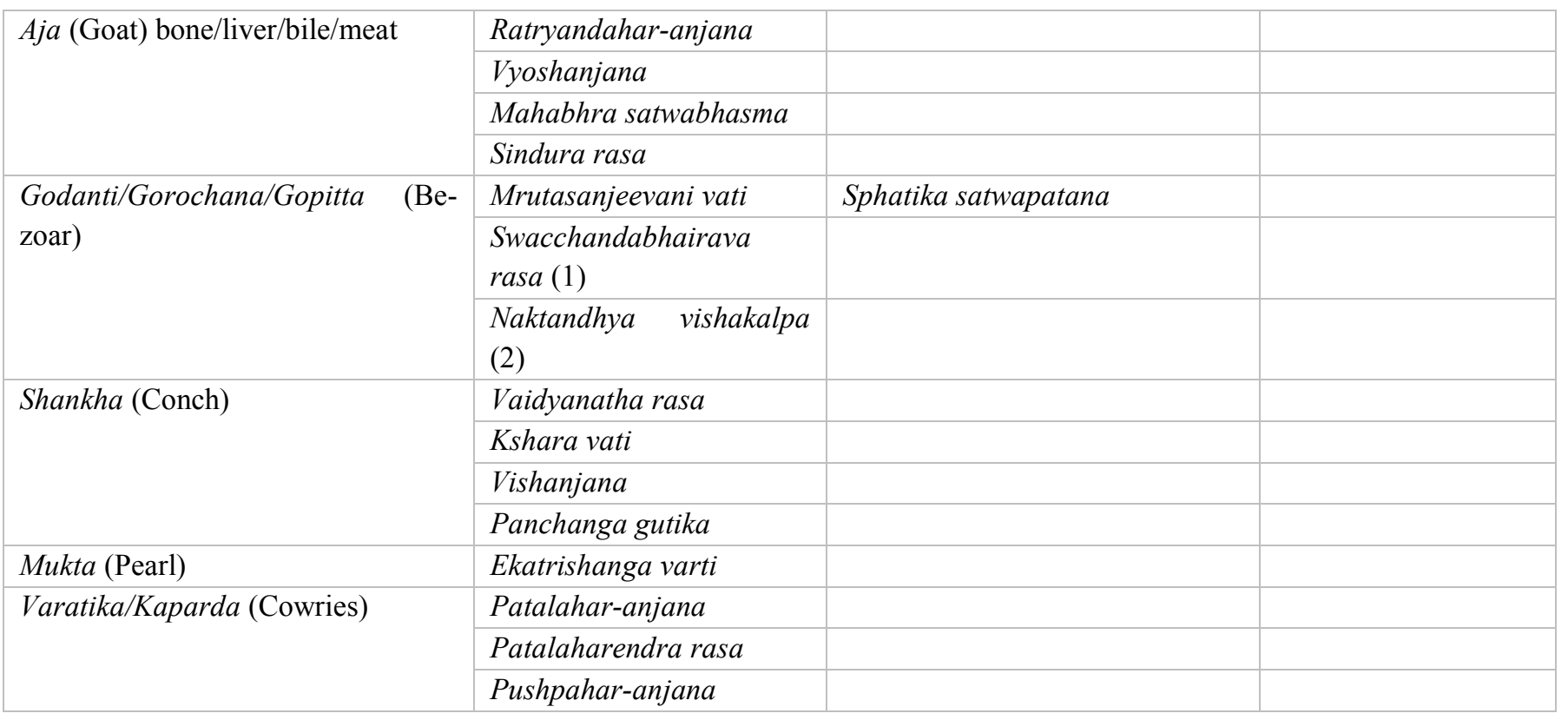

\section{Source of Support: Nil}

\section{Conflict of Interest: None Declared}

How to cite this URL: Devika M.B \& M.S Krishnamurthy: Unusual Animal Resources Explained In Rasa Ratna Samucchaya. International Ayurvedic Medical Journal \{online\} 2020 \{cited August, 2020\} Available from: http://www.iamj.in/posts/images/upload/4190_4198.pdf 